\title{
Effects of High-concentration contrast material and low-voltage CT on contrast for multiphasic CT of the upper abdomen: comparison using the simulation with virtual monochromatic imaging obtained by fast-switch kVp dual-energy CT
}

\author{
Makoto Sakane, Tonsok Kim", Masatoshi Hori, Hiromitsu Onishi, Atsushi Nakamoto, Takahiro Tsuboyama, \\ Mitsuaki Tatsumi and Noriyuki Tomiyama
}

\begin{abstract}
Objective: The purpose of this study was to compare the effects of high-concentration contrast material and low-voltage CT simulated by virtual monochromatic (VM) imaging on contrast enhancement at multiphasic $\mathrm{CT}$ of the upper abdomen.

Methods: This study included 72 patients who underwent $C T$ during early arterial (EAP), late arterial and portal venous phases after 300-mgl/ml (Group A; 34 patients) or 350-mg/ml (Group B; 38 patients) contrast-material injection at the same volumetric rate $(0.067 \mathrm{~mL} / \mathrm{sec} / \mathrm{kg})$. VM images were generated at 50 and $65 \mathrm{keV}$. Contrast-to-noise ratios (CNRs) of aorta, portal vein, and liver parenchyma were calculated and statistically compared.
\end{abstract}

Results: Mean CNRs for 50-keV VM images were significantly higher than 65-keV VM images of each organ at any phases ( $p<0.05)$, except for hepatic parenchyma in EAP. Aortic CNRs in EAP on 65- and 50-keV images of Group B were significantly higher than Group A ( $p<0.05$, respectively). Aortic CNR on 50-keV images of Group A and on 65-keV images of Group B were 11\% and 21\% higher than 65-keV images of Group A, respectively.

Conclusions: Low-voltage CT simulated by VM image improved contrast enhancement through any phases, while high-concentration contrast material increased only arterial contrast in EAP more effectively.

Keywords: High-concentration contrast material; Low-voltage CT; Fast-switch kVp Dual-energy CT;

Virtual monochromatic imaging

\section{Introduction}

Many experiments have been conducted to improve the contrast-to-noise ratio (CNR) of abdominal organs for contrast-enhanced computed tomography (CECT) with reduced radiation exposure (Dawson 2004; Fenchel et al. 2004). The administration protocol of iodinated contrast material for dynamic CT, including total iodine dose, iodine concentration, injection rate, and injection duration, have a significant effect on diagnostic performance especially of upper abdominal and vascular diseases,

\footnotetext{
* Correspondence: kim@radiol.med.osaka-u.ac.jp

Department of Radiology, Osaka University Graduate School of Medicine, D1, 2-2, Yamadaoka, Suita, Osaka 565-0871, Japan
}

therefore getting so that attainment of higher CNR for organs became of increasing importance (Hänninen et al. 2000; Tsurusaki et al. 2004). The results of numerous investigations consistently demonstrated that administration of higher-concentration contrast materials and a faster bolus injection could lead to higher iodine-dose rate and volumetric rate, causing an increase of effective enhancement and CNR for abdominal organs (Awai et al. 2004; Cademartiri et al. 2005)

Low-tube-voltage CT at 80 or $100 \mathrm{kVp}$ can also improve contrast of vascular and tumor enhancement and applied to abdominal CT, because X-ray generated with low voltage CT is markedly absorbed by high-atomic 
number substances such as iodinated contrast material (Takahashi et al. 1998; Ravenel et al. 2001; Funama et al. 2005; Nakayama et al. 2005). Recently, fast-switch $\mathrm{kVp}$ dual-energy CT using the new technology of fast tubevoltage switching has been developed, with which a virtual monochromatic (VM) image can be reconstructed at any desired monochromatic energy from 40 to 140 kiloelectron voltages (keV) (Wu et al. 2009). Its application makes it possible to create multiple VM images for different voltages reconstructed from one set of dualenergy projection data and to make simulated assessment of those images under the same conditions. Some reports have reported that administration of high-concentration contrast material and low-keV VM image can both improve the CNR for abdominal contrast-enhanced CT, none of them have so far compared the relationship between these two factors under the same conditions (Kulkarni et al. 2012). We hypothesized that the impact of low-voltage CT on contrast enhancement of abdominal organs may be greater than that of high-concentration contrast material during any dynamic phases.

Low-voltage $80 \mathrm{kVp}$ and usual-voltage $120 \mathrm{kVp}$ conventional CT images can be simulated by VM images of two different energies ( $50 \mathrm{keV}$ and $65 \mathrm{keV}$ ) obtained with fast-switch $\mathrm{kVp}$ dual-energy CT, respectively (Wu et al. 2009). In this study, we evaluated CNRs for multiphasic contrast-enhanced CT of aorta, portal vein, and hepatic parenchyma by using two contrast materials at different concentrations and VM images for the two-different voltage simulation. The purpose of this study was to compare the effects of high-concentration contrast material and low-voltage CT simulated by VM imaging on contrast enhancement at multiphasic $\mathrm{CT}$ of the upper abdomen.

\section{Materials and methods Patient population}

Our institutional review board approved this retrospective study and waived the requirement for informed consent. We searched our medical records of our hospital for patients who underwent initial investigation by multiphasic contrast-enhanced CT of the upper abdomen with dual-energy CT scanner using dual-energy technique for detection of known or suspected liver or pancreas tumors between January 2010 and March 2011 for inclusion of this study. We found such 80 patients, but eight patients were excluded from this study because it was difficult to place the region of interest (ROI) accurately on the organ concerned for measurement of attenuation due to total occlusion of the portal vein $(\mathrm{n}=1)$, major tumor invasion of the liver $(\mathrm{n}=2)$, or too thin a subcutaneous fat layer of the anterior abdominal wall $(n=5)$. As a result, a total of 72 patients were included in this study.
The patients were divided into two groups. Group A consisted of 34 patients who underwent CT examination using the protocol with $300 \mathrm{mgI} / \mathrm{ml}$ contrast material (22 men and 12 women; mean age, 65 years; age range, 20-90 years; mean body weight, $60 \mathrm{~kg}$; weight range, 40-86 kg). Group B consisted of 38 patients who underwent CT examination using the protocol with $350 \mathrm{mgI} / \mathrm{ml}$ contrast material (21 men and 17 women; mean age, 65 years; age range, 33-84 years; mean body weight, $61 \mathrm{~kg}$; weight range, $40-89 \mathrm{~kg}$ ).

Thirty patients were clinically diagnosed with hepatic cirrhosis (Group A: $\mathrm{n}=16$; Group B: $\mathrm{n}=14$ ), and liver tumors were clinically or histopathologically proven in 36 patients ( 25 hepatocellular carcinomas, 6 liver metastases, 3 cholangiocarcinomas and 2 combined hepatocellular-cholangiocellular carcinomas). Eighteen patients were diagnosed by biopsy or radical surgery with pancreatic adenocarcinoma and one patient with carcinoma of the ampulla of Vater. None of the patients showed tumor invasion of the major vessels or obstructive jaundice, which could affect contrast enhancement of the abdominal organs and portal systems (Table 1).

\section{Dual-energy CT technique}

Multiphasic CT was performed with a fast $\mathrm{kVp}$ switching 64-section multi-detector row dual-energy CT scanner (Discovery 750 HD; GE healthcare, Milwaukee, WI). This scanner consists of a single-source CT tube that switches rapidly within a fraction of a millisecond between 80 and $140 \mathrm{kVp}$, so that two complete sets of projection data at 80 and $140 \mathrm{kVp}$ could be collected simultaneously with minimal influence from misregistration artifacts. The tube-current setting was automatically preset at $600 \mathrm{~mA}$ by the software for the dual-energy

Table 1 Patient-group demographics

\begin{tabular}{lcc}
\hline Patients back ground & Group A & Group B \\
\hline Number of patients & 34 & 38 \\
Number of men & 22 & 21 \\
Age $(\mathrm{y})$ & $65(20-90)$ & $65(33-84)$ \\
Weight (kg) & $60(40-86)$ & $61(40-89)$ \\
Clinical condition & & \\
$\quad$ Hepatic cirrhosis & 16 & 14 \\
$\quad$ Hepatocellular carcinoma & 14 & 11 \\
$\quad$ Pancreatic adenocarcinoma & 7 & 600 \\
\hline Contrast material infusion protocols & & 350 \\
\hline Administered iodine dose (mgl/kg) & 600 & 26 \\
lodine concentration (mg/mL) & 300 & 0.067 \\
Injection duration (s) & 30 & 24 \\
Volumetric rate (mL/sec/kg) & 0.067 & 20 \\
lodine-dose rate (mgl/sec/kg) & &
\end{tabular}


CT system. The field of view covered by the detector was $(34.5-40.0) \mathrm{cm}^{2}$ depending on the physical size of the patient. Data were acquired with a detector configuration of $64 \times 0.625 \mathrm{~mm}$, rotation time of 0.5 second and pitch of 1.375 . Collimation of $0.625 \times 64$ slices was chosen for dual energy acquisition. After scanning, $5 \mathrm{~mm}$-thick, conventional $140-\mathrm{kVp}$ CT images were automatically reconstructed for clinical use. The patient dose indexes of the volume CT dose index (CTDIvol) and the dose-length products were recorded from the console of the CT scanner. The CTDIvol value displayed by the scanner was $12.72 \mathrm{mGy}$ for each scan.

\section{Contrast material infusion protocols and multiphasic data acquisition}

After the acquisition of anteroposterior abdominal scout radiographs, the patients underwent multiphasic dualenergy CT scanning comprising early arterial, late arterial and portal venous phase scans. Conventional abdominal CT scanning before the injection of contrast material were obtained in all patients, and additional scanning of the equilibrium phase were performed when clinically indicated. All patients were intravenously injected with 600 $\mathrm{mgI} / \mathrm{kg}$ of total body iodine load of non-ionic contrast material by means of insertion of a 20- or 22-gauge IV catheter into an antecubital vein and a power injector (Dual-Shot Type D; Nemoto Kyorindo, Tokyo, Japan). Two-different injection protocols of contrast material were used: an injection of $300-\mathrm{mgI} / \mathrm{ml}$ concentration contrast material, iomeprol (Iomeron 300; Bracco-Eisai Co., Ltd., Tokyo, Japan) for 30 seconds for Group A, and an injection of $350-\mathrm{mgI} / \mathrm{ml}$ concentration contrast material, iomeprol (Iomeron 350; Bracco-Eisai Co., Ltd.) for $26 \mathrm{sec}-$ onds for Group B. The volumetric rate of the contrast material per patient body weight was the same for the two groups $(0.067 \mathrm{~mL} / \mathrm{sec} / \mathrm{kg}$ for Group A and Group B). On the other hand, the iodine-dose rate per patient body weight was $20 \mathrm{mgI} / \mathrm{sec} / \mathrm{kg}$ for Group A and $24 \mathrm{mgI} / \mathrm{sec} / \mathrm{kg}$ for Group B. Scanning delay after the injection was determined with a semi-automatic computer-assisted bolus tracking program (Smart Prep; GE Healthcare), and early arterial, late arterial, and portal venous phasic CT scanning was started 8, 20 and 50 seconds respectively after the trigger (the threshold level set at an increase of $100 \mathrm{HU}$ over the baseline CT number of the abdominal aorta at the level of the celiac axis by $140-\mathrm{kVp}$ CT image on the console).

\section{Post-processing of dual-energy data}

The dual-energy data thus obtained were sent to a computer workstation (Advantage Windows; GE healthcare) and the $5 \mathrm{~mm}$-thick VM image of each phase was reconstructed on the workstation with analytical software for dual-energy data (GSI viewer; GE healthcare). The paired-projection data collected by dual-energy scan were analyzed in terms of the material decomposition process to determine the material density projection after a series of calibrations and corrective steps. A monochromatic energy image could be generated from the weighted sum of material density projections with their corresponding mass attenuation coefficients at a given energy. For any virtual $\mathrm{keV}$ between 40 and $140 \mathrm{keV}$, the object is depicted on the workstation as if imaged with a monochromatic X-ray beam that simulated $\mathrm{keV}$ (Wu et al. 2009). VM images of two different energies $(50 \mathrm{keV}$ and $65 \mathrm{keV}$ ) were generated as the images that had attenuation properties similar to conventional CT images at $80 \mathrm{kVp}$ and $120 \mathrm{kVp}$, and conventional CT images at $140 \mathrm{kVp}$ were also transferred to the workstation as reference images. As a result, a set of nine images was obtained per patient for one examination: $5-\mathrm{mm}$ thick $140-\mathrm{kVp}$ conventional CT, $65-\mathrm{keV}$ $\mathrm{VM}$ and 50-keV VM images for each of the early arterial, late arterial and portal venous phase.

\section{Quantitative analyses}

Quantitative measurements were performed at the same workstation by a radiologist with 8 years' experienced. A total of nine image sets from one patient were displayed side by side on two screens with a preset abdominal window. For each of the image sets, the attenuations of abdominal aorta, portal vein, hepatic parenchyma, paraspinal muscle and subcutaneous fat tissue in the anterior abdominal wall were measured by manually placing the circular or oval ROI cursor on the organ concerned at a slice level which included the hepatic hilum. The shape, size and position of the ROIs were kept constant with the cut-and-paste function of the workstation. The displacement of abdominal organs among the early arterial, late arterial and portal venous scanning attributable to the patient's continuous breathing caused slight differences in the cursor positions, so that each of the ROIs was carefully confirmed to be placed on the organ of interest and manually adjusted to nearly the same position on all the image sets. Attenuations of each organ were also measured on unenhanced CT.

Attenuation of the abdominal aorta was recorded from a single ROI (mean area, $183 \mathrm{~cm}^{2}$; range, 77-260) that was relatively the same size as the vessel lumen at the level of the celiac trunk. Calcifications and soft plaques were carefully avoided for each ROI. Attenuation of the portal vein was also recorded from a single ROI (mean area, $93 \mathrm{~cm}^{2}$; range, 40-197) at the level of the main portal vein. ROIs as large as the vessel were carefully placed to reduce the effect of inhomogeneous attenuation of the vessel. Attenuation of the hepatic parenchyma was recorded as the average of the CT values of 3 ROIs (mean area, $400 \mathrm{~cm}^{2}$; range, 384-414) placed on 
the anterior and posterior segment and on the left lobe of the liver. Areas containing hepatic tumors and focal changes in the liver, large vessels and prominent artifacts were also avoided. Attenuation of the paraspinal muscle was recorded from a single ROI (mean area, $196 \mathrm{~cm}^{2}$; range, 118-220) without including the macroscopic area of fat infiltration. Image noises were measured for each image set as standard deviations of pixel values from ovoid ROIs (mean area, $201 \mathrm{~cm}^{2}$; range, 180 - 209) drawn on the homogenous region of subcutaneous fat tissue in the anterior abdominal wall.

The contrast-to-noise ratio (CNR) for aorta, portal vein and liver parenchyma in comparison with that for muscle was calculated with the equation: $\mathrm{CNR}=\left(\mathrm{ROI}_{\mathrm{o}}-\right.$ $\left.R O I_{m}\right) / S_{n}$, where $R_{0 I}$ was the attenuation of a given organ, $\mathrm{ROI}_{\mathrm{m}}$ was the attenuation of the paraspinal muscle, and $\mathrm{SD}_{\mathrm{n}}$ was the average standard deviation of subcutaneous fat tissue (or the image noise). In addition, increases in the mean CNRs for 50-keV VM images in Group A, 65-keV VM images in Group B, and 50-keV VM images in Group B over those for the $65-\mathrm{keV}$ VM images in Group A were calculated and defined as ratios of increase in CNR for each phase.

\section{Qualitative analyses}

Image quality of $140-\mathrm{keV}$ conventional $\mathrm{CT}$ and $65-$ and $50 \mathrm{keV}$ VM images was evaluated by two board-certified radiologists with 8 and 21 years of experience in abdominal CT, who were blind to acquisition parameters of each image and clinical background. The images at the anatomical slice level including the hepatic hilum in the early arterial, late arterial, and portal venous phase were selected phase by phase and then randomly serialized on the workstation with the standard abdominal window (window level $50 \mathrm{HU}$, window width $350 \mathrm{HU}$ ). The radiologists independently graded the images for image contrast, image noise and overall image quality. Image contrast and overall image quality of the conventional and VM images were rated on a 4-point scale: 1, unacceptable; 2, acceptable; 3, good; 4, excellent. Image noise as graded similarly: 1 , severe noise present and unacceptable; 2, moderate noise present and interfering; 3, mild noise not interfering with depiction of structures; 4 , no substantial noise. The initial setting of window level and width was set as the standard abdominal window.

\section{Statistical analysis}

Statistical analysis was performed with JMP software version 9.2 (SAS Institute Inc., Cary, NC). P $<0.05$ was considered significant for all statistical analyses. The data for the $140 \mathrm{kVp}$ conventional images were also viewed for reference, but not statistically evaluated.

First, student's $t$ test was used to compare the background factors (patient age, body weight) and attenuation of each organ on unenhanced CT of Groups A and B. Second, intragroup attenuation of aorta, portal vein, liver parenchyma, paraspinal muscle and subcutaneous fat tissue (image noise) for each phase observed on 65 and $50-\mathrm{keV}$ VM images was statistically compared by using the paired $t$ test. The same analyses were performed for comparison of CNRs for each organ (aorta, portal vein and liver parenchyma). Third, intergroup CNRs for each organ were statistically compared for Groups A and B by using student's $t$ test for the corresponding images of the 65- and 50-keV VM images at each phase. Finally, the scores for image quality given by the two radiologists to $65-$ and $50 \mathrm{keV}$ VM images for each group were statistically compared by student's $t$ test.

\section{Results}

\section{Background-factors evaluation}

There were no significant differences in patient age and body weight between Groups A and B. No significant differences were observed in clinical findings of liver cirrhosis. Mean attenuation on unenhanced CT of Groups $\mathrm{A}$ and $\mathrm{B}$ were $42.7 \mathrm{HU} \pm 5.1$ (mean \pm standard deviation) and $43.7 \mathrm{HU} \pm 4.5$ for the abdominal aorta, $35.9 \mathrm{HU} \pm$ 7.9 and $37.8 \mathrm{HU} \pm 7.7$ for the portal vein, and $58.1 \mathrm{HU} \pm$ 7.6 and $55.7 \mathrm{HU} \pm 7.4$ for the hepatic parenchyma, respectively. There were no significant differences observed in attenuation of each organ on unenhanced $C T$ between Groups A and B ( $p>0.05)$.

\section{Quantitative analysis}

\section{Attenuation and Image noise}

Mean attenuations for aorta, portal vein, hepatic parenchyma and paraspinal muscle and image noise on 65 and $50-\mathrm{keV}$ VM images together with those for $140-\mathrm{keV}$ conventional images as reference are shown in Table 2. Mean attenuations for each organ observed on $140-\mathrm{kVp}$ conventional, $65-\mathrm{keV}$ and $50-\mathrm{keV} \mathrm{VM}$ images increased in that order at any phase for both patient groups. Moreover, mean attenuations on 50-keV VM images were significantly higher than those on $65-\mathrm{keV}$ VM images for any organ at any phase for both groups $(\mathrm{p}<0.05$, respectively).

With regard to image noise, mean image noises on $50-\mathrm{keV}$ VM images were significantly higher than that on $65-\mathrm{keV}$ VM images ( $<<0.05$, respectively), which was similar to that on $140-\mathrm{kVp}$ conventional images, at any phase for both groups.

\section{CNR}

Mean CNRs for aorta, portal vein and hepatic parenchyma are shown in Table 3. Mean CNRs for each organ observed on $140-\mathrm{kVp}$ conventional, $65-\mathrm{keV}$ and $50-\mathrm{keV}$ VM images increased in that order, making exceptions of hepatic CNRs in the early arterial phase for both 
Table 2 Mean attenuation values for the aorta, portal vein, hepatic parenchyma and paraspinal muscle (HU) and image noise

\begin{tabular}{|c|c|c|c|c|c|c|c|}
\hline & \multirow[b]{2}{*}{ Phase } & \multicolumn{3}{|c|}{$\begin{array}{c}\text { Group A } \\
\text { Attenuation (HU) }\end{array}$} & \multicolumn{3}{|c|}{$\begin{array}{c}\text { Group B } \\
\text { Attenuation (HU) }\end{array}$} \\
\hline & & $140 \mathrm{kVp}$ & $65 \mathrm{keV}$ & $50 \mathrm{keV}$ & 140 kVp & $65 \mathrm{keV}$ & $50 \mathrm{keV}$ \\
\hline \multirow[t]{3}{*}{ Aorta } & Early arterial & $296 \pm 42.2$ & $422 \pm 65.6$ & $751 \pm 119^{*}$ & $355 \pm 56.2$ & $511 \pm 85.0$ & $907 \pm 156^{*}$ \\
\hline & Late arterial & $302 \pm 54.2$ & $441 \pm 82.9$ & $787 \pm 151^{*}$ & $282 \pm 75.6$ & $398 \pm 93.7$ & $705 \pm 172^{*}$ \\
\hline & Portal venous & $154 \pm 39.0$ & $207 \pm 20.3$ & $368 \pm 63.2^{*}$ & $158 \pm 22.1$ & $219 \pm 23.0$ & $375 \pm 32.5^{*}$ \\
\hline \multirow[t]{3}{*}{ Portal vein } & Early arterial & $77.4 \pm 22.2$ & $94.8 \pm 23.4$ & $152 \pm 39.7^{*}$ & $82.0 \pm 23.4$ & $107 \pm 30.5$ & $175 \pm 53.4^{*}$ \\
\hline & Late arterial & $146 \pm 34.5$ & $206 \pm 45.1$ & $353 \pm 81.6^{*}$ & $170 \pm 36.8$ & $250 \pm 50.4$ & $436 \pm 91.2^{*}$ \\
\hline & Portal venous & $154 \pm 18.9$ & $219 \pm 25.3$ & $378 \pm 43.0^{*}$ & $163 \pm 19.7$ & $233 \pm 23.6$ & $398 \pm 47.3^{*}$ \\
\hline Hepatic & Early arterial & $69.7 \pm 9.75$ & $74.9 \pm 9.09$ & $101 \pm 16.8^{*}$ & $69.0 \pm 9.25$ & $78.1 \pm 10.3$ & $105 \pm 18.1^{*}$ \\
\hline \multirow[t]{2}{*}{ Parenchyma } & Late arterial & $89.7 \pm 16.4$ & $141 \pm 16.8$ & $219 \pm 26.3^{*}$ & $91.8 \pm 13.6$ & $145 \pm 13.2$ & $225 \pm 22.9^{*}$ \\
\hline & Portal venous & $113 \pm 10.1$ & $141 \pm 14.4$ & $221 \pm 25.2^{*}$ & $115 \pm 10.3$ & $140 \pm 15.0$ & $217 \pm 24.9^{*}$ \\
\hline Paraspinal & Early arterial & $58.2 \pm 4.98$ & $64.5 \pm 5.07$ & $84.2 \pm 8.18^{*}$ & $57.5 \pm 4.67$ & $64.8 \pm 7.15$ & $85.3 \pm 11.9^{*}$ \\
\hline \multirow[t]{3}{*}{ Muscle } & Late arterial & $61.3 \pm 7.77$ & $67.9 \pm 7.77$ & $89.4 \pm 11.1^{*}$ & $68.8 \pm 7.10$ & $68.8 \pm 7.10$ & $92.0 \pm 12.7^{*}$ \\
\hline & Portal venous & $66.6 \pm 6.09$ & $73.9 \pm 6.23$ & $101 \pm 9.76^{*}$ & $65.0 \pm 5.62$ & $74.6 \pm 7.25$ & $99.3 \pm 18.8^{*}$ \\
\hline & & \multicolumn{3}{|c|}{ Image noise } & \multicolumn{3}{|c|}{ Image noise } \\
\hline \multirow[t]{3}{*}{ Image noise } & Early arterial & $8.27 \pm 1.30$ & $8.06 \pm 1.81$ & $13.1 \pm 1.81^{*}$ & $8.56 \pm 1.50$ & $8.42 \pm 1.84$ & $13.6 \pm 2.03^{*}$ \\
\hline & Late arterial & $8.52 \pm 1.38$ & $8.24 \pm 1.64$ & $13.3 \pm 2.08^{*}$ & $8.84 \pm 1.76$ & $8.91 \pm 2.11$ & $13.4 \pm 2.23^{*}$ \\
\hline & Portal venous & $8.57 \pm 1.39$ & $8.44 \pm 1.57$ & $13.5 \pm 1.84^{*}$ & $8.96 \pm 1.92$ & $8.61 \pm 2.05$ & $13.8 \pm 1.91^{*}$ \\
\hline
\end{tabular}

Data are shown as mean attenuation \pm standard deviation.

*Differences were statistically significant between $65-\mathrm{keV}$ and $50-\mathrm{keV}$ images $(\mathrm{p}<0.05)$.

groups. Mean CNRs on 50-keV VM images were significantly $(\mathrm{p}<0.05)$ higher than those on $65-\mathrm{keV}$ VM image for each organ at any phase for both patient groups, with the exception of hepatic CNRs in the early arterial phase for group A.

Intergroup comparison of mean CNRs for groups $\mathrm{A}$ and $B$ at the same voltage setting showed significant differences only for the aorta in the early and late arterial phases. Aortic CNRs in the early arterial phase on 65 and 50-keV VM images for Group B were significantly ( $p=0.015$ and $<0.01$, respectively) higher than those for Group A. On the contrary, aortic CNRs in the late arterial phase on the $65-\mathrm{keV}$ and $50-\mathrm{keV}$ VM images for Groups B were significantly ( $\mathrm{p}=0.018$ and 0.01 , respectively) lower than those for Group A.

Table 3 Mean CNRs for the aorta, portal vein and hepatic parenchyma

\begin{tabular}{|c|c|c|c|c|c|c|c|c|c|}
\hline & \multirow[b]{3}{*}{ Phase } & \multirow{2}{*}{\multicolumn{3}{|c|}{$\begin{array}{l}\text { Group A } \\
\text { CNR }\end{array}$}} & \multirow{2}{*}{\multicolumn{3}{|c|}{$\begin{array}{c}\text { Group B } \\
\text { CNR }\end{array}$}} & \multicolumn{2}{|c|}{$\begin{array}{c}\text { Intergroup comparison } \\
\text { Group A vs B }\end{array}$} \\
\hline & & & & & & & & $p$ & \\
\hline & & $140 \mathrm{kVp}$ & $65 \mathrm{keV}$ & $50 \mathrm{keV}$ & $140 \mathrm{kVp}$ & $65 \mathrm{keV}$ & $50 \mathrm{keV}$ & $65 \mathrm{keV}$ & $50 \mathrm{keV}$ \\
\hline Aorta & Early arterial phase & $29.5 \pm 7.17$ & $46.6 \pm 12.7$ & $51.7 \pm 13.1^{*}$ & $36.6 \pm 9.92$ & $56.6 \pm 18.0$ & $62.4 \pm 16.7^{*}$ & 0.015 & $<0.01$ \\
\hline & Late arterial phase & $29.1 \pm 8.66$ & $47.5 \pm 14.2$ & $53.7 \pm 14.8^{*}$ & $25.3 \pm 10.0$ & $38.4 \pm 16.8$ & $44.1 \pm 16.0^{*}$ & 0.018 & 0.01 \\
\hline & Portal venous phase & $10.6 \pm 5.02$ & $16.4 \pm 4.22$ & $20.1 \pm 5.74^{*}$ & $10.8 \pm 3.96$ & $16.6 \pm 5.28$ & $19.6 \pm 4.74^{*}$ & n.s. & n.s. \\
\hline Portal vein & Early arterial phase & $2.41 \pm 2.78$ & $3.88 \pm 3.02$ & $5.31 \pm 3.17^{*}$ & $2.48 \pm 3.60$ & $5.49 \pm 4.29$ & $6.88 \pm 4.20^{*}$ & n.s. & n.s. \\
\hline & Late arterial phase & $10.3 \pm 4.89$ & $19.0 \pm 4.73$ & $22.0 \pm 4.57^{*}$ & $13.0 \pm 5.17$ & $18.9 \pm 5.11$ & $21.8 \pm 4.77^{*}$ & n.s. & n.s. \\
\hline & Portal venous phase & $10.6 \pm 2.93$ & $17.8 \pm 4.59$ & $20.7 \pm 4.38^{*}$ & $11.5 \pm 3.51$ & $18.1 \pm 5.38$ & $21.4 \pm 6.31^{*}$ & n.s. & n.s. \\
\hline Hepatic parenchyma & Early arterial phase & $1.47 \pm 1.29$ & $1.35 \pm 1.45$ & $1.36 \pm 1.48$ & $1.17 \pm 1.87$ & $1.66 \pm 1.80$ & $1.55 \pm 1.94$ & n.s. & n.s. \\
\hline & Late arterial phase & $3.37 \pm 2.05$ & $9.32 \pm 3.16$ & $9.94 \pm 2.67^{*}$ & $3.70 \pm 1.92$ & $8.73 \pm 2.61$ & $9.47 \pm 2.57^{*}$ & n.s. & n.s. \\
\hline & Portal venous phase & $5.69 \pm 1.60$ & $8.28 \pm 2.46$ & $8.96 \pm 2.25^{*}$ & $5.97 \pm 1.92$ & $7.56 \pm 2.77$ & $8.45 \pm 2.83^{*}$ & n.s. & n.s. \\
\hline
\end{tabular}

Data are shown as mean \pm standard deviation.

*Differences were statistically significant between $65-\mathrm{keV}$ and $50-\mathrm{keV}$ images $(p<0.05)$.

n.s.: not significant. 


\section{Ratios for increase in CNR}

The ratios for increase in CNR compared to 65-keV VM images of Group A are shown in Table 4. The aortic ratios on $50-\mathrm{keV}$ VM images of Group A and 65- or 50-keV VM images of Group B showed 1.11, 1.21 and 1.34 CNR increase in the early arterial phase, respectively.

\section{Qualitative analysis}

Mean scores for evaluation of image quality with respect to image contrast, image noise, or overall image quality are shown in Table 5. The scores for image contrast on $50-\mathrm{keV}$ VM images in the early arterial phase image were noticeably better than those for those on $65-\mathrm{keV}$ VM images for both patient groups, although the scores for image noise and overall image quality were worse (Figures 1 and 2).

\section{Discussion}

Low-voltage CT of the abdomen has been gaining the attention of investigators because it has the potential to improve contrast enhance for hypervascular liver lesions in the late arterial phase with less contrast material and a lower radiation dose (Nakayama et al. 2005; Altenbernd et al. 2011). It is also reported to be useful for improving enhancement of the pancreas and peripancreatic vasculature or improvement of tumor conspicuity during the pancreatic parenchymal and portal venous phases (Altenbernd et al. 2011; Marin et al. 2010). Moreover, low-voltage CT is used to improve the depiction of anatomically fine structure like peripheral or coronary arteries for the arterial phase and urinary system opacification for excretory phase CT urography (Utsunomiya et al. 2010; Shinagare et al. 2011). Most previous studies of low-voltage CT investigated contrast enhancement during a single phase (Nakayama et al. 2005; Altenbernd et al. 2011; Shinagare et al. 2011) and few studies have investigated contrast for multi-phasic CT. Besides, the use of high-

Table 4 Ratios of increase in CNR on each VM image compared to $65-\mathrm{keV}$ VM image with $300-\mathrm{mgl} / \mathrm{ml}$ concentration contrast material as baseline CNR

\begin{tabular}{|c|c|c|c|c|}
\hline & Phase & $\begin{array}{c}\text { Group A } \\
50 \mathrm{keV}\end{array}$ & $\begin{array}{c}\text { Group B } \\
65 \mathrm{keV}\end{array}$ & $50 \mathrm{keV}$ \\
\hline \multirow[t]{3}{*}{ Aorta } & Early arterial & 1.11 & 1.21 & 1.34 \\
\hline & Late arterial & 1.13 & 0.81 & 0.93 \\
\hline & Portal venous & 1.23 & 1.01 & 1.20 \\
\hline \multirow[t]{3}{*}{ Portal vein } & Early arterial & 1.37 & 1.41 & 1.77 \\
\hline & Late arterial & 1.16 & 0.99 & 1.15 \\
\hline & Portal venous & 1.16 & 1.02 & 1.20 \\
\hline Hepatic & Early arterial & 1.01 & 1.26 & 1.14 \\
\hline \multirow[t]{2}{*}{ Parenchyma } & Late arterial & 1.07 & 0.94 & 1.02 \\
\hline & Portal venous & 1.08 & 0.91 & 1.02 \\
\hline
\end{tabular}

concentration contrast material has been found to yield superior vascular enhancement compared with moderate-concentration contrast material and not to improve contrast enhancement of liver parenchyma on portal phase and late phase images (Awai et al. 2004; Holalkere et al. 2011; Fleischmann 2003). To the best of our knowledge, no studies have compared the effects of high-concentration contrast material and low-voltage CT or low-voltage VM image on contrast for multiphasic CT of the upper abdomen.

In our study, these effects were evaluated by the simulation using VM images which have become available with the recently developed CT technique of fast-switch $\mathrm{kVp}$ dual-energy scanning (Wu et al. 2009). In our institution, dual-energy CT is clinically used for the diagnosis of hepatic and pancreatic tumors based on the clinical utility reported previously, and the radiation dose for dual-energy CT used in this study was almost similar to that for conventional CT (Heye et al. 2012). VM imaging can depict an object as if imaged with a monochromatic beam at any simulated energy from 40 to $140 \mathrm{keV}$. We employed VM images reconstructed at two different photon energies of $65-$ and $50-\mathrm{keV}$ on the basis of previously reported findings. The first of these findings was that a comparison between $65-\mathrm{keV} \mathrm{VM}$ images and conventional $\mathrm{CT}$ images found equivalent performances. In particular, the CNR of VM images at around $65-\mathrm{keV}$ was similar or slightly better to that on conventional 120-kVp CT images (Zhang et al. 2011; Yu et al. 2011). The second, Huda et al. demonstrated that the X-ray tube potentials of 80 and $120 \mathrm{kVp}$ were similar to the representative photon energies of 52 and $66 \mathrm{keV}$, respectively (Huda et al. 2000). The third was that 120$\mathrm{kVp}$ was the tube voltage in general clinical use for conventional $\mathrm{CT}$, while $80-\mathrm{kVp}$ was commonly used voltage for low-voltage CT of the abdomen (Altenbernd et al. 2011; Marin et al. 2010). Patients had to be scanned twice to compare intra-patient attenuation differences on conventional single-energy CT images at low- and high-tube voltages, while the efficacy of VM image was evaluated by comparing image sets reconstructed at two-different $\mathrm{keV}$ from one clinical dual-energy data. It has also been reported that VM imaging improved image quality compared to conventional CT due to a reduction in beam-hardening artifacts (Wu et al. 2009; Matsumoto et al. 2011). A phantom study showed that image noise on VM images for the range of 67$72 \mathrm{keV}$ was significantly lower than that on $120-\mathrm{kVp}$ conventional CT images, with CNR on the VM image highest at $68 \mathrm{keV}$ (Matsumoto et al. 2011). Before starting our study, we considered that those characteristics of VM imaging might make it possible to compare the same clinical data under conditions of better image quality. 
Table 5 Mean visual scores for image contrast, image noise and overall image quality

\begin{tabular}{|c|c|c|c|c|c|c|c|c|}
\hline & & & & Group A & & & Group B & \\
\hline & & & & Score & & & Score & \\
\hline & Phase & & $140 \mathrm{kVp}$ & $65 \mathrm{keV}$ & $50 \mathrm{keV}$ & $140 \mathrm{kVp}$ & $65 \mathrm{keV}$ & $50 \mathrm{keV}$ \\
\hline Image contrast & Early arterial & Reader 1 & $3.1 \pm 0.3$ & $3.4 \pm 0.4$ & $3.9 \pm 0.2^{*}$ & $3.2 \pm 0.4$ & $3.4 \pm 0.4$ & $3.9 \pm 0.2^{*}$ \\
\hline & & Reader 2 & $3.3 \pm 0.4$ & $3.5 \pm 0.5$ & $3.8 \pm 0.3^{*}$ & $3.4 \pm 0.5$ & $3.5 \pm 0.5$ & $3.8 \pm 0.3^{*}$ \\
\hline & Late arterial & Reader 1 & $3.3 \pm 0.4$ & $3.8 \pm 0.3$ & $3.9 \pm 0.2$ & $3.5 \pm 0.5$ & $3.9 \pm 0.1$ & $4.0 \pm 0.0$ \\
\hline & & Reader 2 & $3.2 \pm 0.5$ & $3.8 \pm 0.3$ & $3.9 \pm 0.2$ & $3.5 \pm 0.5$ & $3.9 \pm 0.2$ & $4.0 \pm 0.0$ \\
\hline & Portal venous & Reader 1 & $3.4 \pm 0.5$ & $3.8 \pm 0.3$ & $3.9 \pm 0.1$ & $3.4 \pm 0.5$ & $3.9 \pm 0.1$ & $3.9 \pm 0.2$ \\
\hline & & Reader 2 & $3.3 \pm 0.5$ & $3.8 \pm 0.3$ & $4.0 \pm 0.0$ & $3.4 \pm 0.4$ & $3.8 \pm 0.3$ & $3.9 \pm 0.1$ \\
\hline Image noise & Early arterial & Reader 1 & $3.7 \pm 0.4$ & $3.5 \pm 0.4$ & $2.9 \pm 0.5^{*}$ & $3.7 \pm 0.4$ & $3.4 \pm 0.5$ & $2.9 \pm 0.4^{*}$ \\
\hline & & Reader 2 & $3.9 \pm 0.2$ & $3.8 \pm 0.7$ & $3.3 \pm 0.6^{*}$ & $3.9 \pm 0.2$ & $3.6 \pm 0.5$ & $3.3 \pm 0.7$ \\
\hline & Late arterial & Reader 1 & $3.5 \pm 0.5$ & $3.2 \pm 0.6$ & $2.7 \pm 0.7^{*}$ & $3.6 \pm 0.4$ & $3.1 \pm 0.7$ & $2.8 \pm 0.6$ \\
\hline & & Reader 2 & $4.0 \pm 0.0$ & $3.7 \pm 0.8$ & $3.4 \pm 0.8$ & $3.9 \pm 0.2$ & $3.8 \pm 0.7$ & $3.2 \pm 0.9$ \\
\hline & Portal venous & Reader 1 & $3.4 \pm 0.5$ & $3.3 \pm 0.6$ & $3.2 \pm 0.6$ & $3.7 \pm 0.4$ & $3.3 \pm 0.5$ & $3.2 \pm 0.4$ \\
\hline & & Reader 2 & $3.9 \pm 0.2$ & $3.6 \pm 0.4$ & $3.5 \pm 0.5$ & $4.0 \pm 0.0$ & $3.7 \pm 0.4$ & $3.5 \pm 0.5$ \\
\hline Overall image quality & Early arterial & Reader 1 & $3.7 \pm 0.4$ & $3.4 \pm 0.4$ & $3.0 \pm 0.3^{*}$ & $3.6 \pm 0.4$ & $3.5 \pm 0.5$ & $2.9 \pm 0.3^{*}$ \\
\hline & & Reader 2 & $3.4 \pm 0.5$ & $3.5 \pm 0.4$ & $3.2 \pm 0.6$ & $3.5 \pm 0.5$ & $3.2 \pm 0.8$ & $3.2 \pm 0.8$ \\
\hline & Late arterial & Reader 1 & $3.2 \pm 0.4$ & $3.2 \pm 0.6$ & $2.9 \pm 0.6^{*}$ & $3.5 \pm 0.5$ & $3.2 \pm 0.6$ & $2.9 \pm 0.6^{*}$ \\
\hline & & Reader 2 & $3.2 \pm 0.5$ & $3.6 \pm 0.8$ & $3.2 \pm 0.6$ & $3.5 \pm 0.5$ & $3.7 \pm 0.7$ & $3.2 \pm 0.9$ \\
\hline & Portal venous & Reader 1 & $3.5 \pm 0.5$ & $3.5 \pm 0.5$ & $3.4 \pm 0.5$ & $3.7 \pm 0.4$ & $3.5 \pm 0.5$ & $3.4 \pm 0.5$ \\
\hline & & Reader 2 & $3.3 \pm 0.4$ & $3.5 \pm 0.5$ & $3.5 \pm 0.5$ & $3.5 \pm 0.5$ & $3.7 \pm 0.4$ & $3.5 \pm 0.5$ \\
\hline
\end{tabular}

Data are shown as mean \pm standard deviation.

*Differences were statistically significant between $65-\mathrm{keV}$ and $50-\mathrm{keV}$ images $(p<0.05)$.

n.s.: not significant.

Our results demonstrated that low-keV VM images improved aortic and portal venous CNRs in any phases and hepatic CNRs in the late arterial and portal venous phases. In the patient group injected with $300-\mathrm{mgI} / \mathrm{ml}$ concentration contrast material, $50-\mathrm{keV} \mathrm{VM}$ images showed $11-23 \%$ increase for the aorta and $16-37 \%$ for the portal vein in each phase, and $7-8 \%$ increase in CNR for the liver parenchyma in the late arterial and portal venous phases in comparison with the results of $65-\mathrm{keV}$ VM images. These results indicate that $50-\mathrm{keV}$
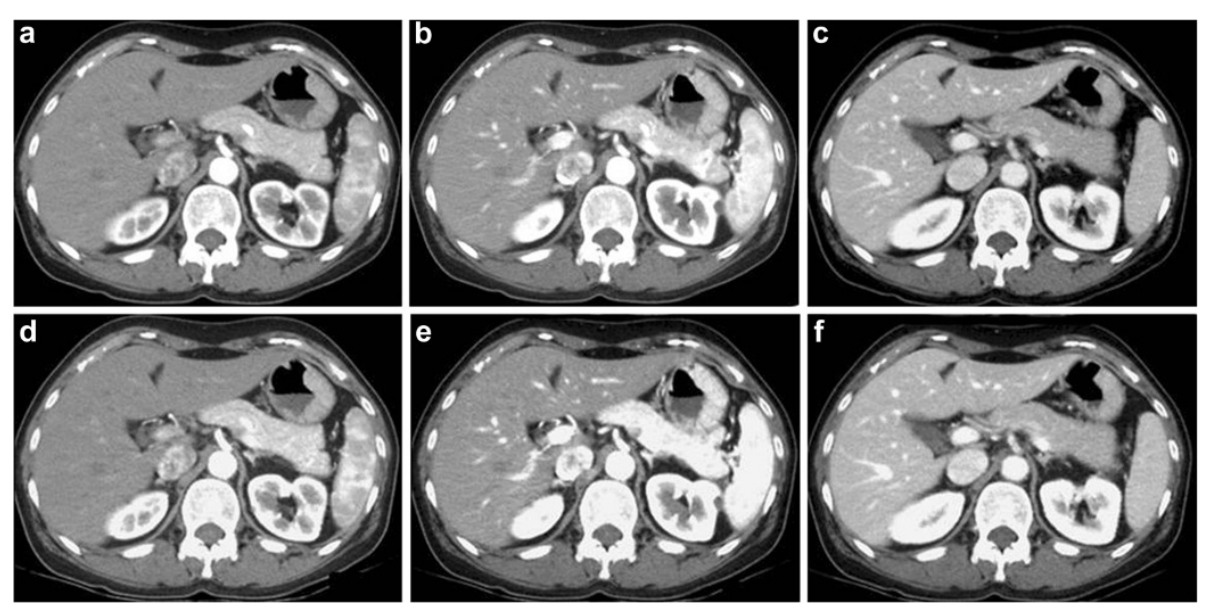

Figure 1 60-year-old weighed 50 kg and had a normal liver. $\mathbf{a}$, b, and $\mathbf{c}$ : 65-keV VM images; $\mathbf{d}$, e, and $\mathbf{f}$ : 50-keV VM images obtained in the early arterial ( $\mathbf{a}$ and $\mathbf{d})$ ), late arterial (b and e), and portal venous (c and $\mathbf{f})$ phases after administration of $600 \mathrm{mgl} / \mathrm{kg}$ of 300-mgl/ml concentration contrast material, which were displayed with the same preset abdominal window (window width, $350 \mathrm{HU}$; window level, $50 \mathrm{HU}$ ). Attenuation of vessels and hepatic parenchyma is increased on the 50-keV VM images compared with the 65-keV VM images. 

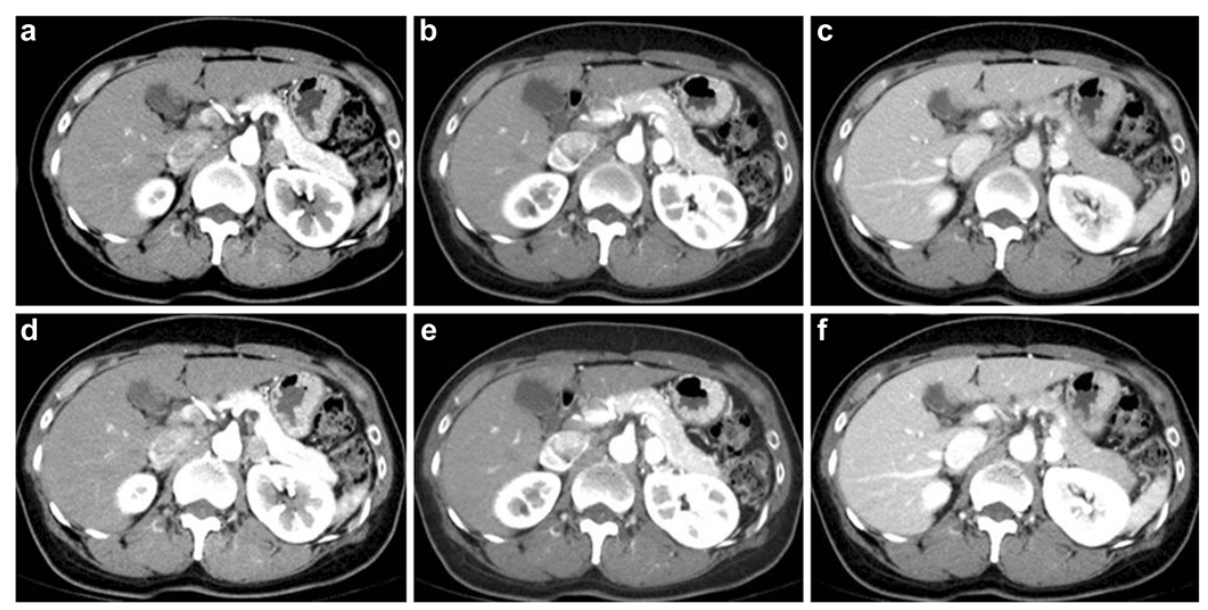

Figure 2 53-year-old woman weighed $\mathbf{5 0} \mathbf{~ k g}$ and had a normal liver. $\mathbf{a}$, b, and $\mathbf{c}$ : 65-keV VM images; $\mathbf{d}$, e, and f: 50-keV VM images obtained

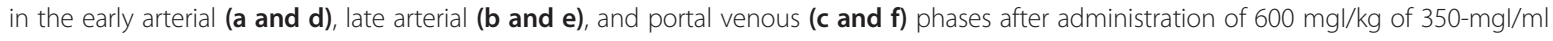
concentration contrast material, which were displayed with the standard abdominal window. Attenuation of vessels and hepatic parenchyma is increased on the 50-keV VM images compared with the 65-keV VM images.

VM imaging can improve abdominal CNR in any phase, even when the image niose was increased by the lower $\mathrm{keV}$ reconstruction (Lewis et al. 2013). Our results also demonstrated that use of high-concentration contrast material can increase the aortic enhancement significantly in the early arterial phase. As aortic CNRs on 50$\mathrm{keV}$ VM images for Group A and on 65-keV VM image for Group B increased by $11 \%$ and $21 \%$ over CNR for the $65-\mathrm{keV}$ VM images for Group A respectively, greater improvement in aortic CNR in the early arterial phase could be achieved with the use of high-concentration contrast material compared to the findings for $50-\mathrm{keV}$ VM images. Use of high concentration, which enables a higher iodine delivery rate without increasing injection speed, is thought to be more advantageous than lowvoltage $\mathrm{CT}$ for $\mathrm{CT}$ arteriography in patients with heavy weight, in which image quality deteriorates as a result of increased image noise with low-voltage CT. Moreover, the CNR ratio for the aorta in the early arterial phase on 50-keV VM images for Group B increased by 34\% compared to baseline CNR of $65-\mathrm{keV}$ VM images for Group A. These findings suggested that we can choose an appropriate combination of a $\mathrm{keV}$ setting and an iodine concentration independently to improve image quality for a specific phase imaging. For example, 1) a combined use of low-keV VM image and high-concentration contrast material can be effective for improving arterial enhancement, and 2) lesion-to-surrounding tissue contrast of either hypervascular or hypovascular tumors would probably be increased with low-keV VM image in the late arterial and portal venous phase.

There have been a few clinical reports focusing on the effectiveness of abdominal VM images reconstructed at low $\mathrm{keV}$ and appropriate weighing factor of dual-source dual energy CT (Kulkarni et al. 2012; Maturen et al. 2012; Kim et al. 2010; Sun et al. 2012). Generally, lowvoltage CT is supposed to result in deterioration of image quality as a result of increased image noise especially in patients with weighing $70 \mathrm{~kg}$ or more because the quantity of penetrating photons must decrease substantially at the same level of radiation exposure (Sun et al. 2012). The increase in image noise associated with low-voltage CT can be partially offset by an increase in the tube-current-time product with a reduction in total radiation. As was expected in our study, noise level on $50-\mathrm{keV}$ VM images was higher than on $65-\mathrm{keV}$ VM images. The results of visual evaluation indicated that image qualities of $50-\mathrm{keV} \mathrm{VM}$ images were acceptable, although further study is needed to evaluate the practical utility of low-voltage VM imaging for the abdomen. There were several potential limitations to our study. First, this was a retrospective study with a small number of patients with a variety of backgrounds. A further prospective trial is thus needed to validate our preliminary results. Second, no saline chaser was used after injection of contrast material, although previous studies had demonstrated that a saline chaser was effective to enhance the contrast for abdominal organs and to reduce the total dose of contrast material (Dorio et al. 2003). When a certain volume of high-concentration contrast material was trapped in a capacitance vein, it could be interpreted as more iodine having remained in the dead space. It might be possible to underestimate image contrast when using high-concentration contrast material. Third, simulation of low-voltage CT using VM imaging was utilized in this study. Noise level of low-keV VM image may not be same as that of low-kVp CT with same dose. Therefore, further study using low-kVp CT is necessary. 
Fourth, we could not evaluate either the visualization of peripheral arteries at $\mathrm{CT}$ arteriography, or diagnostic value of hyper- or hypo-vascular tumors because of the low prevalence of malignant tumors in our study population.

To summarize, low-keV VM image improved contrast enhancement for vasculature and hepatic parenchyma in any phases of multi-phasic upper-abdominal CT, while use of high-concentration contrast material improved only arterial contrast in the early arterial phase. A combination of low-keV VM imaging and high-concentration contrast material administration was effective for improving arterial enhancement.

\section{Competing interests}

The authors declare that they have no competing interests.

\section{Authors' contributions}

MS was responsible for the conception and design of the study, contributed to collection of data, performed analysis and interpretation of data, drafted the article and was the guarantor for the paper. TK was responsible for the conception and design of the study, contributed to collection of data, perform analysis and interpretation of data, coordinated the study and critically edited the manuscript. $\mathrm{MH}$ contributed to collection of data and critically edited the manuscript. $\mathrm{HO}, \mathrm{AN}, \mathrm{TT}$ and MT contributed to collection of data. NT helped in the conception and design of the study. Finally, all authors approved the manuscript.

\section{Received: 12 March 2014 Accepted: 17 April 2014}

Published: 8 May 2014

\section{References}

Altenbernd J, Heusner TA, Ringelstein A, Ladd SC, Forsting M, Antoch G (2011) Dual-energy-CT of hypervascular liver lesions in patients with HCC: investigation of image quality and sensitivity. Eur Radiol 21(4):738-743

Awai K, Inoue M, Yagyu Y, Watanabe M, Sano T, Nin S, Koike R, Nishimura Y, Yamashita $Y$ (2004) Moderate versus high concentration of contrast material for aortic and hepatic enhancement and tumor-to-liver contrast at multi-detector row CT. Radiol 233(3):682-688

Cademartiri F, Mollet NR, van der Lugt A, McFadden EP, Stijnen T, de Feyter PJ, Krestin GP (2005) Intravenous contrast material administration at helical 16-detector row CT coronary angiography: effect of iodine concentration on vascular attenuation. Radiol 236(2):661-665

Dawson P (2004) Multi-slice CT, contrast enhancement regimens. Clin Radiol 59 (12):1051-1060

Dorio PJ, Lee FT, Henseler KP, Pilot M, Pozniak MA, Winter TC, Shock SA (2003) Using a saline chaser to decrease contrast media in abdominal CT. AJR Am J Roentgenol 180(4):929-934

Fenchel S, Fleiter TR, Aschoff AJ, van Gessel R, Brambs HJ, Merkle EM (2004) Effect of iodine concentration of contrast media on contrast enhancement in multislice CT of the pancreas. Br J Radiol 77(922):821-830

Fleischmann D (2003) MDCT of renal and mesenteric vessels. Eur Radiol 13(Suppl 5):M94-M101

Funama Y, Awai K, Nakayama Y, Kakei K, Nagasue N, Shimamura M, Sato N, Sultana S, Morishita S, Yamashita Y (2005) Radiation dose reduction without degradation of low-contrast detectability at abdominal multisection $\mathrm{CT}$ with a low-tube voltage technique: phantom study. Radiol 237(3):905-910

Hänninen EL, Vogl TJ, Felfe R, Pegios W, Balzer J, Clauss W, Felix R (2000) Detection of focal liver lesions at biphasic spiral $C$ : randomized double-blind study of the effect of iodine concentration in contrast materials. Radiol 216(2):403-409

Heye T, Nelson RC, Ho LM, Marin D, Boll DT (2012) Dual-energy CT applications in the abdomen. AJR Am J Roentgenol 199(5 Suppl):S64-S70

Holalkere NS, Matthes K, Kalva SP, Brugge WR, Sahani DV (2011) 64-Slice multidetector row $\mathrm{CT}$ angiography of the abdomen: comparison of low versus high concentration iodinated contrast media in a porcine model. Br J Radiol 84(999):221-228

Huda W, Scalzetti EM, Levin G (2000) Technique factors and image quality as functions of patient weight at abdominal CT. Radiology 217(2):430-435
Kim KS, Lee JM, Kim SH, Kim KW, Kim SJ, Cho SH, Han JK, Choi BI (2010) Image fusion in dual energy computed tomography for detection of hypervascular liver hepatocellular carcinoma: phantom and preliminary studies. Invest Radiol 45(3):149-157

Kulkarni NM, Sahani DV, Desai GS, Kalva SP (2012) Indirect computed tomography venography of the lower extremities using single-source dual-energy computed tomography: advantage of low-kiloelectron volt monochromatic images. J Vasc Interv Radiol 23(7):879-886

Lewis M, Reid K, Toms AP (2013) Reducing the effects of metal artefact using high keV monoenergetic reconstruction of dual energy CT (DECT) in hip replacements. Skeletal Radiol 42(2):275-282

Marin D, Nelson RC, Barnhart H, Schindera ST, Ho LM, Jaffe TA, Yoshizumi TT, Youngblood R, Samei E (2010) Detection of pancreatic tumors, image quality, and radiation dose during the pancreatic parenchymal phase: effect of a low-tube-voltage, high-tube-current CT technique-preliminary results. Radiology 256(2):450-459

Matsumoto K, Jinzaki M, Tanami Y, Ueno A, Yamada M, Kuribayashi S (2011) Virtual monochromatic spectral imaging with fast kilovoltage switching: improved image quality as compared with that obtained with conventional 120-kVp CT. Radiology 259(1):257-262

Maturen KE, Kaza RK, Liu PS, Quint LE, Khalatbari SH, Platt JF (2012) "Sweet spot" for endoleak detection: optimizing contrast to noise using low keV reconstructions from fast-switch kVp dual-energy CT. J Comput Assist Tomogr 36(1):83-87

Nakayama Y, Awai K, Funama Y, Hatemura M, Imuta M, Nakaura T, Ryu D, Morishita S, Sultana S, Sato N, Yamashita Y (2005) Abdominal CT with low tube voltage: preliminary observations about radiation dose, contrast enhancement, image quality, and noise. Radiol 237(3):945-951

Ravenel JG, Scalzetti EM, Huda W, Garrisi W (2001) Radiation exposure and image quality in chest CT examinations. AJR Am J Roentgenol 177(2):279-284

Shinagare AB, Sahni VA, Sadow CA, Erturk SM, Silverman SG (2011) Feasibility of low-tube-voltage excretory phase images during $C T$ urography: assessment using a dual-energy CT scanner. AJR Am J Roentgenol 197(5):1146-1151

Sun G, Ding J, Lu Y, Li M, Li L, Li GY, Zhang XP (2012) Comparison of standard- and low-tube voltage 320-detector row volume $\mathrm{CT}$ angiography in detection of intracranial aneurysms with digital subtraction angiography as gold standard. Acad Radiol 19(3):281-288

Takahashi M, Maguire WM, Ashtari M, Khan A, Papp Z, Alberico R, Campbell W, Eacobacci T, Herman PG (1998) Low-dose spiral computed tomography of the thorax: comparison with the standard-dose technique. Invest Radiol 33(2):68-73

Tsurusaki M, Sugimoto K, Fujii M, Sugimura K (2004) Multi-detector row helical CT of the liver: quantitative assessment of iodine concentration of intravenous contrast material on multiphasic $C T$-a prospective randomized study. Radiat Med 22(4):239-245

Utsunomiya D, Oda S, Funama Y, Awai K, Nakaura T, Yanaga Y, Hirai T, Yamashita Y (2010) Comparison of standard- and low-tube voltage MDCT angiography in patients with peripheral arterial disease. Eur Radiol 20(11):2758-2765

Wu X, Langan DA, Xu D, Benson TM, Pack JD, Schmitz AM, Tkaczyk EJ, Leverentz J, Licato P (2009) Monochromatic CT image representation via fast switching dual kVp. Proc SPIE 7258:725845

Yu L, Christner JA, Leng S, Wang J, Fletcher JG, McCollough CH (2011) Virtual monochromatic imaging in dual-source dual-energy $C T$ : radiation dose and image quality. Med Phys 38(12):6371-6379

Zhang D, Li X, Liu B (2011) Objective characterization of GE discovery CT750 HD scanner: gemstone spectral imaging mode. Med Phys 38(3):1178-1188

\section{doi:10.1186/2193-1801-3-234}

Cite this article as: Sakane et al:: Effects of High-concentration contrast material and low-voltage CT on contrast for multiphasic CT of the upper abdomen: comparison using the simulation with virtual monochromatic imaging obtained by fast-switch kVp dual-energy CT. SpringerPlus 2014 3:234. 